\title{
Abnormal subendocardial function in restrictive left ventricular disease
}

Michael Y Henein, Derek G Gibson

Cardiac Department, The Royal Brompton National Heart and Lung Hospital, London

$M$ Y Henein

D G Gibson

Correspondence to: Dr Derek G Gibson, Cardiac Department, The Royal Brompton National Heart and Lung Hospital, Sydney Street, London SW3 $6 \mathrm{NP}$

Accepted for publication 9 February 1994

\begin{abstract}
Objective-To study possible disturbances in left ventricular long axis function in patients with a restrictive filling pattern.

Design-Prospective examination of the left ventricular transverse and longitudinal axes, transmitral flow, and the apexcardiogram.
\end{abstract}

Setting-A tertiary referral centre for cardiac diseases.

Subjects-21 normal subjects, age (SD) 51(11); 30 patients of similar age with a restrictive left ventricular filling pattern, defined as short early diastolic deceleration time less than the lower $95 \%$ confidence limit of the normal value $(120 \mathrm{~ms})$. 20 patients had a normal and 10 had an increased left ventricular end diastolic cavity size.

Results-Mitral Doppler echocardiography: E wave velocity was high only in patients with a normal cavity size. A wave velocity was greatly reduced in the two groups $(P<0.001)$ so that the $E / A$ ratio was abnormally high. The relative $A$ wave amplitude on the apexcardiogram was greatly increased in the two groups: 46(15)\% (mean (SD)) and 54(4)\% v 15(5)\%. Minor axis: Fractional shortening was reduced from $30(10) \%$ to $17(7) \%$ in patients with normal cavity size and to $13(4 \cdot 2) \%$ in those with a dilated cavity $(P$ $<0.001)$, as was the posterior wall thickening fraction from $100(30) \%$ to $42(20) \%$ and $50(25) \%$ respectively $(P<0.001)$. Total systolic epicardial motion was normal and isovolumic relaxation time was short in the two groups. Long axis: Left ventricular abnormalities included reduced total amplitude of motion and its component during atrial systole $(P<$ 0.001 for the two groups at both sites). Peak long axis shortening and lengthening were decreased at both left ventricular sites $(P<0.001)$. The time intervals from $q$ wave of the electrocardiogram and $A_{2}$ (aortic valve closure) to the onset of shortening and lengthening respectively were increased (both $P<0.001)$. Right ventricular long axis function was similarly affected but to a lesser extent.

Conclusion-Left ventricular long axis function is consistently abnormal in patients with restrictive disease whether or not cavity size is increased. Not only are the extent and peak velocity of short- ening reduced, but during diastole the peak early diastolic lengthening rate and amplitude during atrial systole are impaired. Early diastolic long axis motion is asynchronous with respect to transmitral flow and left ventricular minor axis. These effects will impair the overall left ventricular systolic and diastolic function independently of any decrease in passive cavity compliance. Unlike fibrosis, these long axis abnormalities are potentially amenable to treatment.

(Br Heart f 1994;72:237-242)

Ventricular restriction is usually considered to be a disorder of the endocardium or myocardium which spares systolic function, but which reduces passive ventricular compliance enough to compromise filling. ${ }^{1}$ One of its most prominent features is endomyocardial fibrosis, which is specifically recognised as occurring in restrictive cardiomyopathy in the World Health Organization and International Society and Federation of Cardiology definition and classification of the cardiomyopathies. $^{2}$ The aim of this study was to investigate the possibility that this endomyocardial fibrosis might impair left ventricular subendocardial function in such patients. This would have consequences in both systole and diastole, ${ }^{3}$ which should be separable from a simple reduction in passive compliance, and which might contribute significantly to the overall pathophysiology of the disorder.

\section{Patients and methods}

PATIENTS

We studied 30 patients with a restrictive left ventricular filling pattern, in whom a clinical diagnosis of heart failure had been made. Their mean (SD) age was 55(13) years, and five were women. A restrictive filling pattern was defined as an early diastolic mitral flow deceleration time shorter than the lower $95 \%$ confidence limit of the normal value, $120 \mathrm{~ms}$, as measured by pulsed Doppler echocardiography. ${ }^{4}$ Patients with echocardiographic features of amyloid were deliberately excluded. No patient had Loeffler's disease, ChurgStrauss syndrome, hypereosinophilia, endomyocardial fibrosis, or haemochromatosis. Patients were divided into two groups according to their left ventricular end diastolic dimension obtained from the $\mathrm{M}$ mode record- 
ing of the left ventricular minor axis. Twenty patients had a left ventricular cavity size less than the upper $95 \%$ confidence limit of normal, $5.8 \mathrm{~cm}$; two had had aortic valve homograft replacement; one had aortic valve stenosis; coronary artery disease was present in two; hypertension in two; and the other patients (13) had a diagnosis of congestive heart failure of unknown cause. The remaining 10 patients had a large ventricular cavity, with an end diastolic dimension of $6 \mathrm{~cm}$ or more; six had a documented history of ischaemic heart disease and the rest had unexplained congestive cardiac failure. Two patients had a family history of sudden cardiac death. None of the patients had evidence of pericardial disease. Eight patients were in atrial fibrillation and the rest in sinus rhythm. The results from these patients were compared with those from 21 normal controls of mean (SD) age 51(11) years, none of whom had any history of cardiac disease, hypertension, or diabetes.

\section{METHODS}

Mitral flow velocities were recorded from the apical four chamber view with pulsed Doppler echocardiography with either a HewlettPackard or a Doptek system with a $2 \mathrm{MHz}$ transducer, and were recorded with frequency shift calibration.

$M$ mode and cross sectional echocardiograms were recorded using a Hewlett-Packard model 77020 A Sonos 1000 echograph with a $2.5 \mathrm{MHz}$ transducer for the minor and long axes with the patient lying in the left lateral position. $M$ mode echocardiograms of the left ventricular minor axis were recorded from the long axis parasternal view with the cursor at the tips of mitral valve leaflets. Long axis measurements were taken from the apical four chamber view with the cursor at the left, septal, and right sites of the atrioventricular rings.

Electrocardiograms were recorded using a Hewlett-Packard 12 lead electrocardiograph with a filter of $0.05-100 \mathrm{~Hz}$. The PR interval and QRS duration were computed using built in software.

Apexcardiograms were obtained with a Cambridge pulse transducer (time constant four seconds). This was possible in 17 patients from the position of a well defined palpable apex. All recordings were made separately on a Hewlett-Packard or a Honeywell (Echoline 22) strip chart recorder at a paper speed of $100 \mathrm{~mm} / \mathrm{s}$ with a simultaneous electrocardiogram and phonocardiogram. In patients with atrial fibrillation measurements were taken from beats with a cycle length equal within $50 \mathrm{~ms}$. Minor and long axis echocardiograms were digitised and analysed. ${ }^{5}$

\section{MEASUREMENTS}

All values are based on the mean (SD) of three beats.

\section{DOPPLER}

From the mitral pulsed Doppler trace we measured early and late diastolic peak flow velocities and derived the $E / A$ ratio in patients with well identified $E$ and $A$ waves. The early diastolic acceleration time was taken from the onset of the $\mathrm{E}$ wave to its peak, and the deceleration time from the peak to its end. We measured time intervals from $A_{2}$ of the phonocardiogram to the onset and to the peak of the $E$ wave.

\section{MODE}

From the left ventricular minor axis traces we measured end diastolic and end systolic dimensions at the onset of the $q$ wave and $A_{2}$ (the first high frequency component of the second heart sound) respectively, and thus derived fractional shortening. The amplitude of epicardial motion as well as the change in posterior wall thickness between end diastole and end systole were measured, and the thickening fraction was derived as the increase in thickness divided by end diastolic thickness, expressed as a percentage. The isovolumic relaxation time was taken as the interval between $A_{2}$ and the onset of mitral cusp separation in early diastole. The peak rate of minor axis dimension change was derived by digitisation. ${ }^{5}$ The left atrial diameter was measured from the $M$ mode trace of the aortic root.

From the original long axis traces we measured the following at the left, septal, and right sites: the time intervals from the $q$ wave of the electrocardiogram to the onset of long axis shortening and from $\mathrm{A}_{2}$ of the phonocardiogram to the onset of long axis lengthening, as well as the overall amplitude of excursion and its component in late diastole due to atrial contraction. ${ }^{6}$ Peak rates of shortening and lengthening were derived by digitisation. ${ }^{5}$

On the apexcardiogram, the A wave excursion was measured as a percentage of the total amplitude of pulse wave excursion.

From the electrocardiogram recorded by a Hewlett-Packard machine the PR interval and QRS duration were measured from the built in software.

\section{STATISTICAL ANALYSIS}

The tables give individual mean (SD) values. We used an unpaired $t$ test to compare mean values from normal subjects with those from patients in the two groups and also to compare the two patient groups.

\section{Results}

Tables 1-4 show the results obtained.

\section{DOPPLER ECHOCARDIOGRAM}

The mitral $\mathrm{E}$ wave deceleration time was less than $120 \mathrm{~ms}$, the lower $95 \%$ confidence limit of normal, in all 30 patients, by definition, whether or not cavity size was increased (table 1). Its mean (SD) value was $95(15) \mathrm{ms}$ and $95(10) \mathrm{ms}$ in the two groups respectively compared with the normal value of $160(20)$ $\mathrm{ms}$. The peak $\mathrm{E}$ wave velocity was normal in patients with a dilated left ventricular cavity and increased in those with normal end diastolic cavity size. Peak A wave velocity was 
Table 1 Doppler and apexcardiogram characteristics. Values are mean (SD)

\begin{tabular}{|c|c|c|c|}
\hline \multirow[b]{2}{*}{ Variables } & \multirow[b]{2}{*}{ Normal } & \multicolumn{2}{|c|}{ Restrictive lefi ventricle filling } \\
\hline & & $\begin{array}{l}\text { Normal size } \\
(n=20)\end{array}$ & $\begin{array}{l}\text { Dilated } \\
(n=10)\end{array}$ \\
\hline $\begin{array}{l}\text { Mitral flow } \\
\text { Peak E wave (m/s) } \\
\text { Acceleration time (ms) } \\
\text { Deceleration time (ms) } \\
\text { Peak A wave (m/s) } \\
\text { E/A ratio } \\
\mathrm{A}_{2} \text { to onset of E wave (ms) } \\
\mathrm{A}_{2} \text { to peak E wave (ms) } \\
\text { Apexcardiogram }\end{array}$ & $\begin{array}{c}0 \cdot 7(0 \cdot 1) \\
75(10) \\
160(20) \\
0 \cdot 5(0 \cdot 1) \\
1 \cdot 4(0 \cdot 4) \\
85(15) \\
165(30)\end{array}$ & $\begin{array}{c}0.9(0 \cdot 16)^{\star} \\
50(30)^{\star} \\
95(15)^{\star} \\
0 \cdot 14(0 \cdot 1)^{\star} \\
5 \cdot 3(2 \cdot 3)^{\star} \\
70(20) \dagger \\
130(30)^{\star}\end{array}$ & $\begin{array}{c}0 \cdot 7(0 \cdot 17) \dagger \ddagger \\
70(20) \dagger \ddagger \\
95(10)^{\star} \\
0 \cdot 1(0 \cdot 1)^{\star} \\
4 \cdot 9(1 \cdot 3)^{\star} \\
60(30)^{\star} \\
130(10)^{\star}\end{array}$ \\
\hline Percentage $A$ wave to total & $15(5)$ & $54(4)^{\star}$ & $46(15)^{\star} \ddagger \S$ \\
\hline
\end{tabular}

$\star P<0.001 v$ controls.

$+\mathrm{P}<0.01 v$ controls.

+Normal size restricted $v$ dilated restricted.

$\$ \mathrm{P}<0.05$.

A

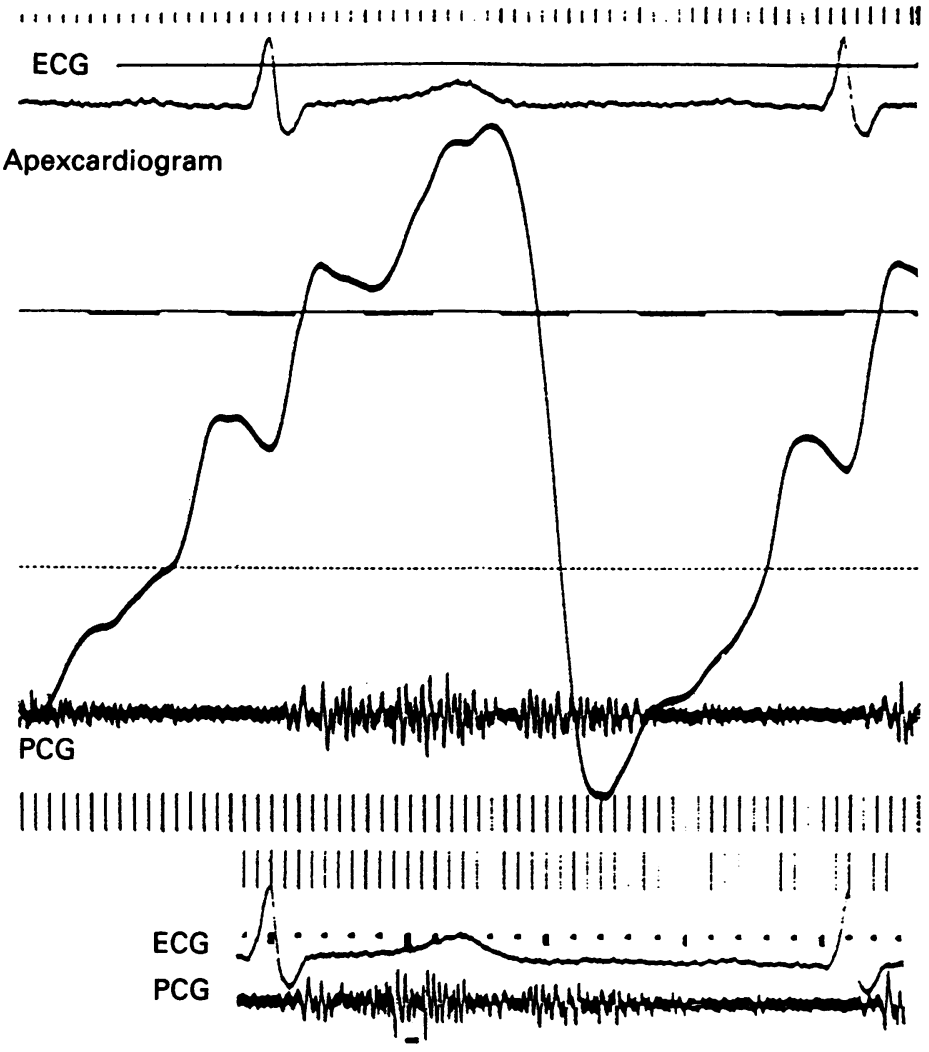

Mitral Doppler

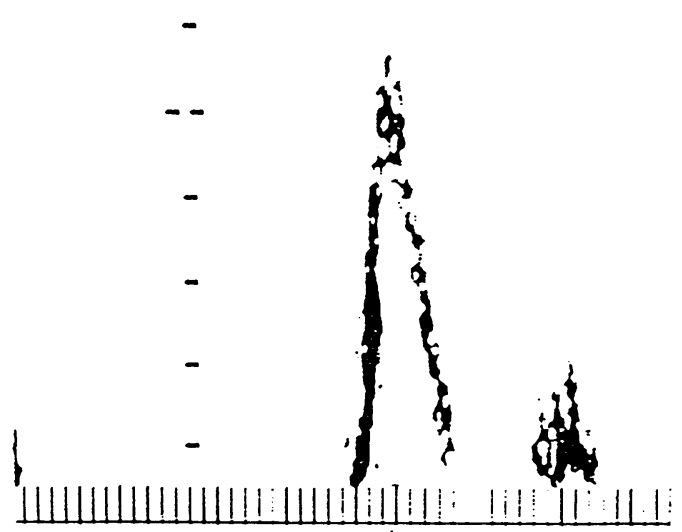

Figure 1 (A) Apexcardiogram from a patient with restrictive left ventricular filling. (B) Mitral flow pattern from the same patient. Note the large $A$ wave pressure increase in the apexcardiogram corresponding to the small Doppler $A$ wave. ECG, Electrocardiogram; and $P C G$, phonocardiogram. reduced in the two groups in patients in sinus rhythm, so the E/A ratio was abnormally high. The $\mathrm{E}$ wave acceleration rate was high only in patients with an increased end diastolic dimension.

The PR interval and QRS duration on the electrocardiogram were normal in the two groups of patients.

\section{APEXCARDIOGRAM}

In patients with sinus rhythm, the relative $A$ wave excursion was considerably higher than normal in the two groups (table 1), particularly in patients with a normal end diastolic cavity dimension (fig 1).

M MODE

Total epicardial motion of the minor axis (table 2) was normal in the two groups but fractional shortening was equally reduced whether or not the end diastolic dimension was increased; the same applied for posterior wall thickening. The isovolumic relaxation time was shorter than normal in the two groups. Peak shortening and lengthening rates were significantly reduced only in patients with normal end diastolic cavity size. The left atrium was dilated in the two groups.

Long axis function (tables 3 and 4) was similarly affected in the two groups. The total amplitude of motion and its late diastolic atrial component were significantly reduced at all sites with the exception of the $A$ wave on the right side, which remained normal in patients with a normal end diastolic dimension. Peak long axis shortening and lengthening rates were also decreased at all sites, again with the exception of the right side, which was normal in patients with dilated ventricles. The time intervals from the $\mathrm{q}$ wave of the electrocardiogram to the onset of long axis shortening and from $A_{2}$ of the phonocardiogram to the onset of lengthening were abnormally increased in the two groups and at all sites including the right side.

In normal subjects, the minor axis starts to lengthen before blood flow can be detected across the mitral valve. The long axis behaves similarly, starting to lengthen $27(7) \mathrm{ms}$ and 25(5) $\mathrm{ms}$ at the left and septal sites respectively before the earliest detectable mitral flow. In the two patient groups, however, the onset of long axis lengthening followed that of early diastolic flow by $15-35 \mathrm{~ms}$ and $5-15 \mathrm{~ms}$ $(P<0.001)$ respectively at the two sites (fig 2).

\section{Discussion}

Although the diagnosis of restrictive left ventricular disease has traditionally been made invasively, ${ }^{1}$ mitral deceleration time is consistently reduced in this disorder to less than the lower $95 \%$ confidence limit of normal. ${ }^{4} \mathrm{We}$ used this criterion to identify 20 such patients who originally presented with clinical evidence of heart failure. We excluded those with amyloid disease, as these have been previously studied in detail. ${ }^{7}$ As reported elsewhere, ${ }^{8}$ the most common clinical association was with 
Table 2 Minor axis dimensions and velocities. Values are mean (SD)

\begin{tabular}{|c|c|c|c|}
\hline \multirow[b]{2}{*}{ Variables } & \multirow[b]{2}{*}{ Normal } & \multicolumn{2}{|c|}{ Restrictive lefi ventricular filling } \\
\hline & & $\begin{array}{l}\text { Normal size } \\
(n=20)\end{array}$ & $\begin{array}{l}\text { Dilated } \\
(n=10)\end{array}$ \\
\hline \multicolumn{4}{|l|}{ Minor axis } \\
\hline End systolic dimension $(\mathrm{cm})$ & $3.3(0.5)$ & $4.5(0.8)^{\star}$ & $6.4(0.8)^{\star}$ \\
\hline Fractional shortening (\%) & $30(10)$ & $17(7)^{\star}$ & $13(4 \cdot 2)^{\star}$ \\
\hline PW thickening fraction (\%) & $100(30)$ & $42(20)^{\star}$ & $50(25)^{\star}$ \\
\hline Systolic epicardial motion (cm) & $0.9(0.1)$ & $0.7(0.3)$ & $0.85(0.15)$ \\
\hline IVR time (ms) & $55(10)$ & $10(20)^{\star}$ & $20(30)^{\star}$ \\
\hline IVR dimension change $(\mathrm{cm})$ & $0.13(0 \cdot 1)$ & $0.11(0.1)$ & $0 \cdot 13(0 \cdot 1)$ \\
\hline \multicolumn{4}{|l|}{ Peak rate of } \\
\hline Shortening $(\mathrm{cm} / \mathrm{s})$ & $9 \cdot 0(3 \cdot 1)$ & $5.4(2.5)^{\star}$ & $7 \cdot 5(2 \cdot 9)$ \\
\hline $\begin{array}{l}\text { Lengthening }(\mathrm{cm} / \mathrm{s}) \\
\text { Left atrial diameter }(\mathrm{cm})\end{array}$ & $10 \cdot 4(2 \cdot 6)$ & $7.5(3.5) \dagger$ & $10(2 \cdot 8)$ \\
\hline Left atrial diameter $(\mathrm{cm})$ & $3.4(0.3)$ & $4.5(0.6)^{\star}$ & $4 \cdot 7(0 \cdot 4)^{\star}$ \\
\hline \multicolumn{4}{|l|}{ Electrocardiogram } \\
\hline PR interval (ms) & $170(25)$ & $175(25)$ & $180(10)$ \\
\hline QRS duration (ms) & $90(15)$ & $95(15)$ & $110(30)$ \\
\hline
\end{tabular}

PW, Posterior wall; IVR, isovolumic relaxation.

$\star \mathrm{P}<0.001 v$ normal controls.

tP $<0.01 v$ normal controls.

coronary artery disease and mild ventricular hypertrophy, though in a substantial proportion, of patients no obvious underlying cause was found, so these patients could be described as having restrictive cardiomyopathy. We compared these patients with uncomplicated restriction with a second group of 10 patients with a restrictive filling pattern, but in whom the left ventricle was enlarged at end diastole. These patients would not therefore have fitted the orthodox invasive criteria of restriction, though the Doppler approach, being independent of cavity size, allowed them to be recognised. ${ }^{89}$

Left ventricular systolic function was consistently abnormal in uncomplicated restriction even when the end diastolic cavity size was normal. In particular, the decrease in the minor axis during ejection was reduced, so

Table 3 Long axis excursions and velocities. Values are mean (SD)

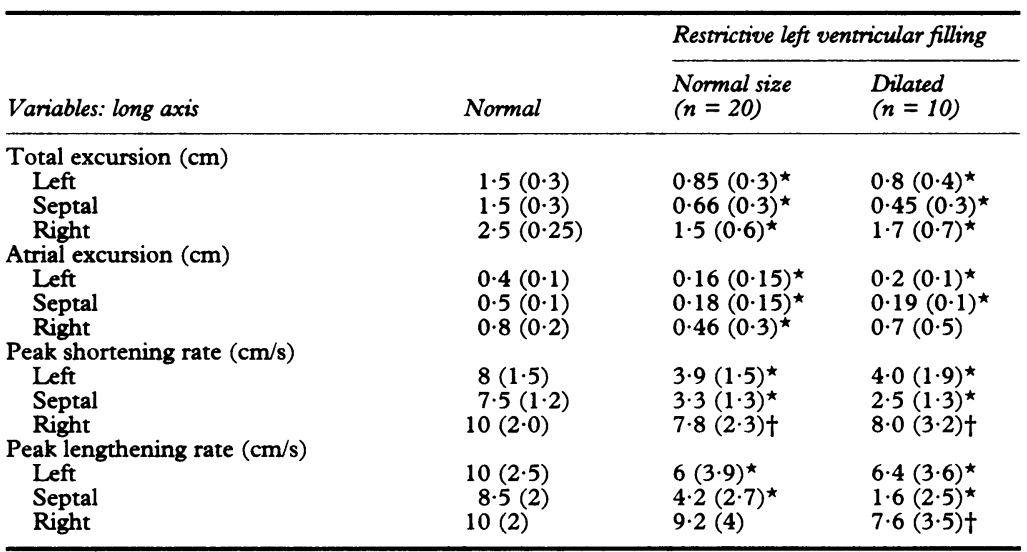

$\star \mathrm{P}<0.001 v$ normal controls

$\dagger \mathrm{P}<0.05 v$ normal controls.

Table 4 Long axis timing. Values are mean (SD)

\begin{tabular}{lllr}
\hline & & \multicolumn{2}{c}{ Restrictive left ventricular filling } \\
\cline { 3 - 4 } Variables & Normal & $\begin{array}{l}\text { Normal size } \\
(n=20)\end{array}$ & $\begin{array}{l}\text { Dilated } \\
(n=10)\end{array}$ \\
\hline $\begin{array}{l}\text { Q to onset of shortening (ms) } \\
\text { Left }\end{array}$ & $90(20)$ & $125(25)^{\star}$ & $140(30)^{\star}$ \\
$\quad \begin{array}{l}\text { Septal } \\
\text { Right }\end{array}$ & $80(10)$ & $125(20)^{\star}$ & $140(50)^{\star}$ \\
$\begin{array}{l}\text { A2 to onset of lengthening (ms) } \\
\text { Left }\end{array}$ & $80(10)$ & $95(15) \dagger$ & $100(25)^{\dagger}$ \\
$\quad$ Septal & $58(11)$ & $75(20)^{\star}$ & $75(15)^{\star}$ \\
Right & $60(9)$ & $85(30)^{\star}$ & $95(30)^{\star}$ \\
\hline${ }^{\star}<0.001 v$ normal controls. & $40(5)$ & $80(25)^{\star}$ & $95(20)^{\star}$ \\
$\dagger P<0.01 v$ normal controls. & & & \\
\hline$P$ & & &
\end{tabular}

that the shortening fraction was consistently low. Minor axis decreases during ejection because of the combined effects of inward motion of the epicardium and an increase in myocardial thickness, particularly that of the posterior wall. ${ }^{3}$ In our patients, the epicardium moved fairly normally during systole, but the extent of posterior wall thickening was consistently reduced.

If the decrease in the minor axis was due entirely to the shortening of circumferentially arranged myocardial fibres, systolic wall thickening would be exactly determined by the inward motion of epicardium, as the myocardium in incompressible. ${ }^{10}$ The extent of thickening in normal subjects is too great to be explained simply by circumferential fibre shortening; it is necessary to invoke the additional contribution of obliquely or longitudinally directed fibres. ${ }^{11}$ The combination of normal epicardial motion and reduced thickening thus strongly suggests that such longitudinal shortening, and hence fattening, is selectively lost in patients with restriction. We confirmed this by showing directly that the extent and peak velocity of longitudinal shortening were reduced. Further, the onset and the termination of long axis shortening were delayed with respect to that of the minor axis. Motion in the two axes could not, therefore, have simply been due to transverse and longitudinal components of homogenous obliquely arranged myocardium, but must have been caused by anatomically separate fibres. As most longitudinally directed fibres are subendocardial, ${ }^{12}$ we suggest that the characteristic systolic abnormality in uncomplicated restriction is the direct effect of selectively impaired subendocardial function.

Diastolic long axis function was also disturbed in uncomplicated restriction. Lengthening did not start until well after transmitral flow had begun; during the period of accelerating blood flow, therefore, long axis fibres were still in late systole (fig 2). The overall amplitude of lengthening during atrial systole was also greatly reduced. Observing the long axis allows an aspect of atrial systole to be studied which is independent of cavity pressure and transmitral flow velocity. The increase in ventricular volume occurring at this stage of the cardiac cycle is accommodated largely by upward motion of the atrioventricular ring, away from the apex of the heart and not by expansion of the minor axis. ${ }^{3}$ As the blood itself does not move with respect to the chest wall, the effects of the atrioventricular ring motion are not reflected in the transmitral Doppler echocardiogram. The reduction in this longitudinal component seen in uncomplicated restriction cannot be ascribed to impaired atrial contraction, as the A wave amplitude on the apexcardiogram was much greater than normal, reflecting a corresponding increase in the pressure $\mathrm{A}$ wave. ${ }^{13}$ The presence of atrial enlargement implies an even greater proportional increase in atrial systolic wall tension. Thus, the combination of an abnormally large A wave on the apexcardiogram and reduced extension of the long 
Figure 2 (A) Mitral Doppler recording from a normal subject (left) and a patient with restrictive filling (right). Vertical scale: one division represents $20 \mathrm{~cm} / \mathrm{s}$. (B) Minor axis recording. (C) Long axis recording from the lefi and septal sites. Vertical scale in centimetres. Note that lengthening normally starts before the onset of transmitral flow (left), but in the patient its onset delayed, particularly at the septal site. Vertical line corresponds to A2; broken line to onset of early diastolic flow. ECG,

Electrocardiogram; PCG, phonocardiogram; and $A_{2}$ aortic valve closure.
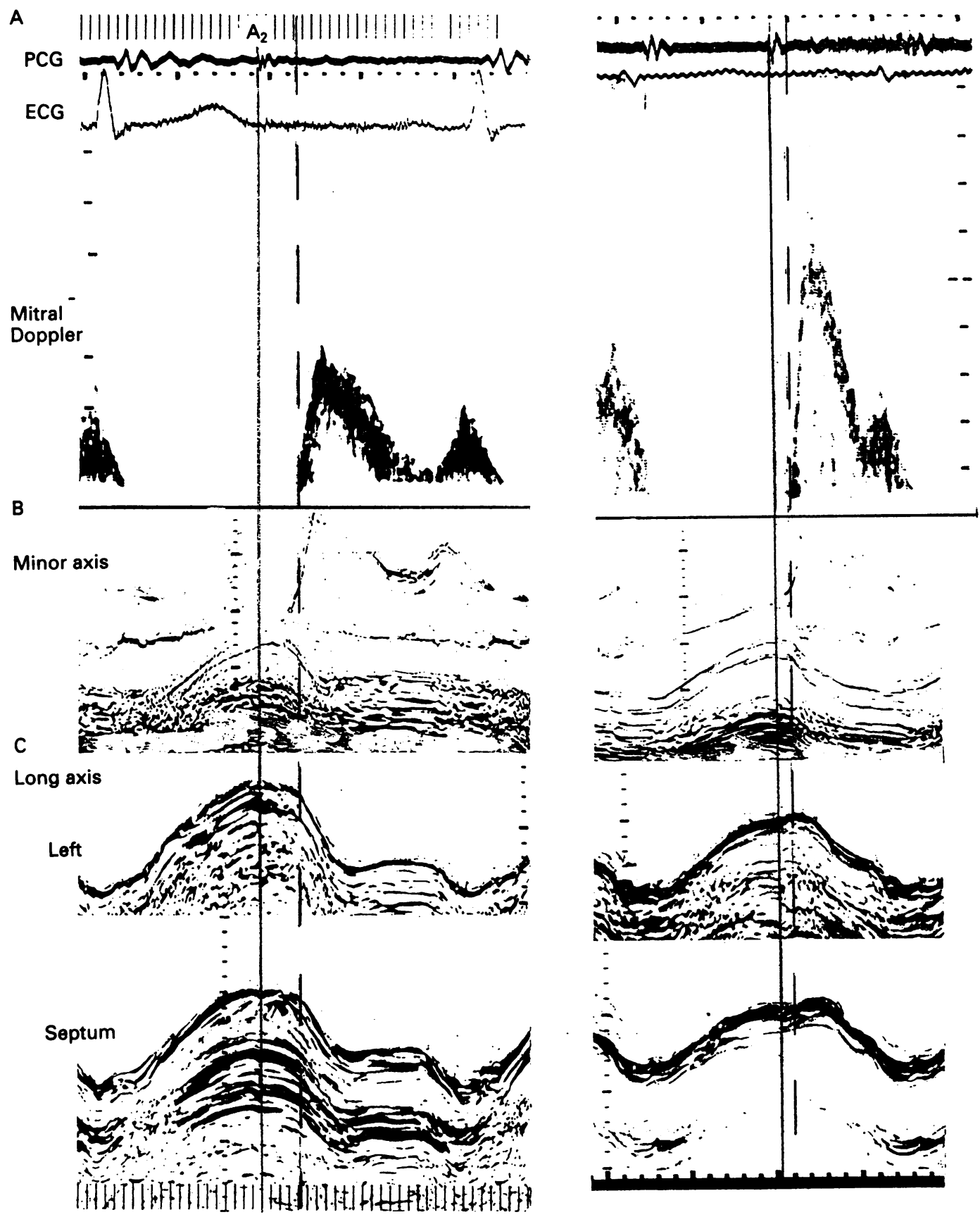

axis (fig 1) shows increased passive stiffness of the left ventricular myocardium in the longitudinal direction.

The disturbances of long axis function in the patients in whom cavity size was increased at end diastole were similar to those in whom it was normal. The overall amplitude of long axis shortening was reduced, and time relations were disturbed in exactly the same way as in patients with uncomplicated restriction. The amplitude of epicardial motion was normal, and so were the peak rates of shortening and lengthening of the ventricular minor axis. During atrial systole, a reduced A wave amplitude on the transmitral Doppler and long axes was again combined with an increase in the pressure A wave. In spite of obvious differences in cavity architecture, therefore, similar long axis abnormalities were associated with a similar disturbance to filling in early and late diastole in the two patient groups. The right ventricle also played a part, though to a lesser extent than the left. The proportional impairment in the overall amplitude of motion and, in particular, of shortening and lengthening velocities, were not as obvious, whereas values for atrial systole were often close to or within the normal range.

We thus found direct and indirect evidence for depressed longitudinal function during side of the heart in patients with a restrictive filling pattern. This was not the effect of abnormal ventricular activation as the $Q R S$ duration was normal. The disturbances in the timing of movement were similar to those in patients with coronary artery disease, ${ }^{6}$ suggesting an ischaemic basis. Some of our patients did have large vessel coronary artery disease, but even in its absence, increased ventricular diastolic pressures predispose to subendocardial ischaemia. ${ }^{14}$ This ischaemia systole and diastole, particularly on the left 
may also underlie the fibrosis which is such a characteristic feature of restrictive ventricular disease.

We believe that these long axis abnormalities may play a part in restrictive disease. Impaired longitudinal shortening is largely responsible for the reduced shortening fraction that was consistently seen in these patients. A major feature of normal early diastolic filling is the change in cavity shape towards a more spherical configuration as the volume increases. ${ }^{15}$ As a sphere has the maximum volume per unit surface area, such a shape change reduces the need for myocardial distension. The left ventricle becomes less spherical at end systole because of preferential shortening of the minor axis. ${ }^{16}$ As myocardial thickening was reduced by impaired long axis function, the reduced long axis shortening will have reduced the shape change, thus increasing the proportion of stroke volume to be accommodated by stretching of the abnormal ventricular wall during the succeeding diastole. Delayed onset of long axis relaxation implies myocardial tension persisting into the period of early diastolic filling, thus increasing myocardial stiffness by a mechanism independent of any change in its passive properties. Reduced changes in cavity shape will add to the effects of the abnormal myocardium in reducing cavity compliance, and may explain it completely in those patients in whom myocardial histology is normal. ${ }^{8}$

Disturbances of early diastolic filling are usually accompanied by an increase in the proportion of the stroke volume entering the ventricle with atrial systole. The reduced extent of longitudinal lengthening, however, the dominant mechanism by which ventricular cavity size increases during atrial systole, impairs this compensating mechanism. Loss of the atrial contribution means that filling must take place almost exclusively in early diastole in spite of major relaxation and geometrical abnormalities. This can be achieved only at the expense of a high left atrial pressure, reflected in our patients by a short isovolumic relaxation time. ${ }^{17}$ Further evidence that impaired long axis function may directly affect early diastole is provided by the effects of intravenous milrinone in patients with cavity dilatation and a restrictive filling pattern. ${ }^{18}$ Its administration causes an increase in the amplitude of mitral ring motion, which is accompanied by increased wall thickening, along with prolongation of isovolumic relax- ation and return of the filling pattern towards normal.

We conclude, therefore, that far from being an incidental finding in restriction, abnormal long axis function is common and contributes significantly to the physiological disturbances so characteristic of the disorder. Although established myocardial fibrosis is probably inaccessible to treatment, long axis abnormalities may be at least potentially reversible, as shown by the effect of milrinone, thus opening an avenue of treatment in these difficult cases.

1 Benotti JR, Grossman W. Restrictive cardiomyopathy. Ann Rev Med 1984;35:113-25.

2 Report of the World Health Organisation and International Society and Federation of Cardiology task force on the definition and classification of cardiomyopathies. Br Heart $\mathcal{F}$ 1980;44:672-3.

3 Jones CJH, Raposo L, Gibson DG. Functional importance of the long axis dynamics of the human left ventricle. $B$ Heart $f$ 1990;63:215-20.

4 Appleton CP, Hatle LK, Popp RL. Demonstration of restrictive ventricular physiology by Doppler echocardiography. $₹$ Am Coll Cardiol 1988;11:757-68.

5 Upton MT, Gibson DG. The study of left ventricular function from digitized echocardiograms. Progr Cardiovasc Dis 1978;20:359-84.

6 Henein MY, Priestley K, Davarashvili T, Buller N, Gibson DG. Early changes in left ventricular subendocardia function after successful coronary angioplasty. $\mathrm{Br} \mathrm{Hear}$ F 1993;69:501-6.

7 Klein AL, Hatle LK, Taliercio CP, et al. Serial Doppler echocardiographic follow-up of left ventricular diastolic function in cardiac amyloidosis. $₹ \mathrm{Am}$ Coll Cardiol 1990;16:1135-41.

8 Keren A, Popp RL. Assignment of patients into the classification of cardiomyopathies. Circulation 1992;86 1622-33

9 Pinamonti B, Di Lenarda A, Sinagra G, et al. Restrictive left ventricular filling pattern in dilated cardiomyopathy eft ventricular assessed by clinical prognostic implications. $\mathcal{f}$ Am Coll Cardiol 1993;22: prognostic

10 Sallin EA. Fibre orientation and ejection fraction in human left ventricle. Biophys $\mathcal{f} 1969 ; 9: 954-64$.

11 Dumesnil JG, Shoucri RM, Laurenceau JL, Turcot J. A mathematical model of the dynamic geometry of the intact left ventricle and its application to clinical data. Circulation 1979;59:1024-31.

12 Greenbaum RA, Ho SY, Gibson DG, Becker AE Anderson RH. Left ventricular fibre architecture in man. Br Heart ₹ 1981;45:248-63.

13 Denef B, Van de Werf F, De Geest H, Kesteloot H Calibrated apexcardiography and assessment of left ventricular dynamics in man. Eur 7 Cardiol 1976; 4(suppl):143-52.

14 Cobbe SM. Coronary blood flow and myocardial ischaemia. In: Julian DG, Camm AJ, Fox KM, et al, eds. Diseases of the heart. 1st ed. London: Bailliere eds. Diseases of the

15 Gibson DG, Brown DJ. Continuous assessment of left ventricular shape in man. Br Heart $₹ 1975 ; 37: 904-10$.

16 Hawthorne EW. Symposium on measurements of left ventricular volume. Part III. Dynamic geometry of the left ventricle. Am f Cardiol 1966;18:566-73.

17 Mattheos M, Shapiro E, Oldershaw PJ, Sacchetti R, Gibson DG. Non-invasive assessment of changes in lef ventricular relaxation by combined phono-, echo-, and mechanocardiography. Br Heart $₹$ 1982;47:253-60.

18 Brecker SJD, Xiao HB, Mbaissouroum M, Gibson DG. Effects of intravenous Milrinone on left ventricular function in ischaemic and idiopathic dilated cardiomyopathy. Am $f$ Cardiol 1993;71:203-9. 